\title{
First moments of the nucleon generalized parton distributions from lattice QCD
}

\section{A. Sternbeck ${ }^{* 1} ;$ M. Göckeler ${ }^{1}$, Ph. Hägler $^{2}$, R. Horsley ${ }^{3}$, Y. Nakamura ${ }^{4}$, A. Nobile ${ }^{5}$, D. Pleiter ${ }^{1,5}$, P.E.L. Rakow ${ }^{6}$, A. Schäfer ${ }^{1}$, G. Schierholz ${ }^{7}$, J. Zanotti ${ }^{8}$}

${ }^{1}$ Institut für Theoretische Physik, Universität Regensburg, 93040 Regensburg, Germany

${ }^{2}$ Institut für Kernphysik, Johannes Gutenberg-Universität Mainz, 55128 Mainz, Germany

${ }^{3}$ School of Physics and Astronomy, University of Edinburgh, Edinburgh EH9 3JZ, UK

${ }^{4}$ RIKEN Advanced Institute for Computational Science, Kobe, Hyogo 650-0047, Japan

5 JSC, Jülich Research Centre, 52425 Jülich, Germany

${ }^{6}$ Theoretical Physics Division, Department of Mathematical Sciences, University of Liverpool, Liverpool L69 3BX, UK

${ }^{7}$ Deutsches Elektronen-Synchrotron DESY, 22603 Hamburg, Germany

${ }^{8}$ School of Chemistry and Physics, University of Adelaide, SA 5005, Australia

E-mail: andre.sternbeckeur.de

We report on our lattice calculations of the nucleon's generalized parton distributions (GPDs), concentrating on their first moments for the case of $N_{f}=2$. Due to recent progress on the numerical side we are able to present results for the generalized form factors at pion masses as low as $260 \mathrm{MeV}$. We perform a fit to one-loop covariant baryon chiral perturbation theory with encouraging results.

The XXIX International Symposium on Lattice Field Theory - Lattice 2011

July 10-16, 2011

Squaw Valley, Lake Tahoe, California

* Speaker.

${ }^{\dagger}$ Supported by the FP7-People Programme of the European Commission. 


\section{Introduction}

The study of the internal structure of hadrons still presents an exciting challenge. Among the different types of studies, the computation of Generalized Parton Distributions (GPDs) is especially challenging, but also attractive, because of their potential for hadron physics.

GPDs were introduced in the late 90s. For a given hadron, they provide detailed information on the partonic structure with respect to spatial, momentum and spin degrees of freedom. GPDs combine the information of the traditional form factors and parton distribution functions (containing them as limiting cases) into a single set of functions and hence contain information also on the correlation between the momentum, spin and spatial degrees of freedom. For the nucleon, one hopes GPDs will provide one day a three-dimensional spatial picture, a better understanding of its spin structure and a value for the quark orbital angular momentum [1].

Beside the renormalization scale ${ }^{1}$, GPDs depend on three kinematic variables: the longitudinal parton momentum fraction $x$, the skewness parameter $\xi$ and the virtuality $t$. The quark structure of a nucleon, for example, is governed by eight GPDs. Among these, the unpolarized GPDs $H$ and $E$ parametrize the off-diagonal matrix element

$$
\left\langle N\left(P^{\prime}\right)\left|\mathscr{O}_{V}^{\mu}(x)\right| N(P)\right\rangle=\bar{U}\left(P^{\prime}\right)\left\{\gamma^{\mu} H(x, \xi, t)+\frac{i \sigma^{\mu v} \Delta_{v}}{2 m_{N}} E(x, \xi, t)\right\} U(P)+\text { higher twist } .
$$

Here $P$ and $P^{\prime}$ denote the incoming and outgoing nucleon momenta (and so $\Delta=P^{\prime}-P, \bar{P}=$ $\left(P^{\prime}+P\right) / 2, t=\Delta^{2}$ and $\left.\xi=-n \cdot \Delta / 2\right)$ and $\mathscr{O}_{V}^{\mu}(x)$ is the light-cone bilocal operator

$$
\mathscr{O}_{V}^{\mu}(x)=\int_{-\infty}^{\infty} \frac{d \lambda}{2 \pi} e^{i \lambda x} \bar{q}\left(-\frac{\lambda}{2} n\right) \gamma^{\mu} \mathscr{P} e^{-i g \int_{\lambda / 2}^{-\lambda / 2} d \alpha n A(\alpha n)} q\left(\frac{\lambda}{2} n\right)
$$

which often arises in hard scattering processes (see, e.g., [3]). $n$ (with $\bar{P} \cdot n=1$ ) denotes a light cone vector in Eq.(1.2) and $\mathscr{P}$ the correct path-ordering of the gluon fields $A$. The polarized nucleon GPDs, $\tilde{H}$ and $\tilde{E}$, are defined in a similar manner, replacing $\gamma^{\mu}$ in Eq.(1.2) by $\gamma^{\mu} \gamma_{5}$.

\section{GPDs and the lattice}

GPDs can be accessed experimentally, for instance, via deeply virtual Compton scattering. The analysis, however, is rather demanding and requires also a partial modeling of the combined $x$-, $\xi$ - and $t$-dependence. Cross-checks to other methods are thus inevitable.

A promising method is given by lattice QCD computations. Although a direct determination of GPDs on the lattice is not possible, their (Mellin) moments

$$
\int_{-1}^{1} d x x^{n-1} H(x, \xi, t), \quad \int_{-1}^{1} d x x^{n-1} E(x, \xi, t), \quad \cdots
$$

are accessible. For a nucleon, for example, these moments can be calculated via matrix elements $\left\langle N\left(P^{\prime}\right)|O| N(P)\right\rangle$ of local operators $O$. For $H$ and $E$ these operators read

$$
O_{V}^{\mu v_{1} \cdot v_{n-1}}(z)=\bar{q}(z) \gamma^{\{\mu} i \overleftrightarrow{D}^{v_{1}} \ldots i \overleftrightarrow{\left.D^{D_{n-1}}\right\}} q(z)-\text { traces }
$$

\footnotetext{
${ }^{1}$ For simplicity, we drop the explicit reference to the renormalization scale $\mu$ in what follows. It is always implicitly understood. Our lattice data below has been nonperturbatively renormalized [2] and is for the $\overline{\mathrm{MS}}$ scheme at $\mu=2 \mathrm{GeV}$.
} 
where $q$ refers to a quark field, $\overleftrightarrow{D} \equiv \vec{D}-\overleftarrow{D}$ to the covariant derivative and $\{\cdots\}$ to a symmetrization of the Lorentz indices. For a definition and further details on the operators needed for the remaining nucleon GPDs, the reader may refer to [4].

Admittedly, the computation of such matrix elements is quite demanding already for $n \geq 2$, and we are not yet in the stage to provide precision results close to the physical point. Nonetheless, such calculations have become more and more feasible in recent years, and hence have attracted interest from within the lattice community $[5,6,7,8,9,10,11]$.

In what follows, we will restrict ourselves to the two nucleon GPDs $H$ and $E$. Their moments are polynomials in $\xi$,

$$
\int_{-1}^{1} d x x^{n-1}\left[\begin{array}{c}
H(x, \xi, t) \\
E(x, \xi, t)
\end{array}\right]=\sum_{k=0}^{[(n-1) / 2]}(2 \xi)^{2 k}\left[\begin{array}{l}
A_{n, 2 k}(t) \\
B_{n, 2 k}(t)
\end{array}\right] \pm \delta_{n, \text { even }}(2 \xi)^{n} C_{n}(t) .
$$

The expansion coefficients $A, B$ and $C$ are real functions of the momentum transfer $t$ (and the renormalization scale $\mu$ ) and are known as the Generalized Form Factors (GFFs) of the nucleon. In this notation, for instance, $A_{10}$ and $B_{10}$ correspond to the electromagnetic form factors [12], and $A_{20}, B_{20}$ and $C_{2}$ parametrize the matrix elements of the energy-momentum tensor $O_{V}^{\mu v}$

$$
\left\langle N\left(P^{\prime}\right)\left|O_{V}^{\mu v}\right| N(P)\right\rangle=\bar{U}\left(P^{\prime}\right)\left\{\gamma^{\{\mu} \bar{P}^{v\}} A_{20}(t)-\frac{i \Delta_{\rho} \sigma^{\rho\{\mu}}{2 m_{N}} \bar{P}^{v\}} B_{20}(t)+\frac{\Delta^{\{\mu} \Delta^{v\}}}{m_{N}} C_{2}(t)\right\} U(P) .
$$

Below we present results for $A_{20}, B_{20}$ and $C_{2}$. They can be extracted from ratios of two- and three-point correlation functions

$$
R\left(t, \tau, p^{\prime}, p\right)=\frac{C_{3}\left(t, \tau, p^{\prime}, p\right)}{C_{2}\left(t, p^{\prime}\right)} \times\left[\frac{C_{2}\left(\tau, p^{\prime}\right) C_{2}\left(t, p^{\prime}\right) C_{2}(t-\tau, p)}{C_{2}(\tau, p) C_{2}(t, p) C_{2}\left(t-\tau, p^{\prime}\right)}\right]^{1 / 2}
$$

which are proportional to $\left\langle N\left(P^{\prime}\right)\left|O_{V}^{\mu v}\right| N(P)\right\rangle$ and constant in the limit $0 \ll \tau \ll t \lesssim T / 2$ (T is the temporal lattice extension). $C_{2}(t, p)$ is the nucleon two-point function with a source at time 0 and sink at time $t$, and $C_{3}\left(t, \tau, p^{\prime}, p\right)$ is the three-point function with an operator insertion at time $\tau$. The latter we calculate employing the sequential source technique.

\section{Results}

Our data for the GFFs is for gauge configurations thermalized using the standard Wilson gauge action and two flavors of clover-improved Wilson fermions. The gauge couplings are $\beta=5.25,5.29$ and 5.40; and the $\kappa$ values are such that pion masses from $1 \mathrm{GeV}$ down to $260 \mathrm{MeV}$ are simulated, where we primarily work with the data in the mass range $260 \mathrm{MeV} \leq m_{\pi} \leq 490 \mathrm{MeV}$. The scale is fixed through setting $r_{0}=0.5 \mathrm{fm}$. This is about the value we obtain from chiral extrapolations of our nucleon mass data [13] for the same set of config-

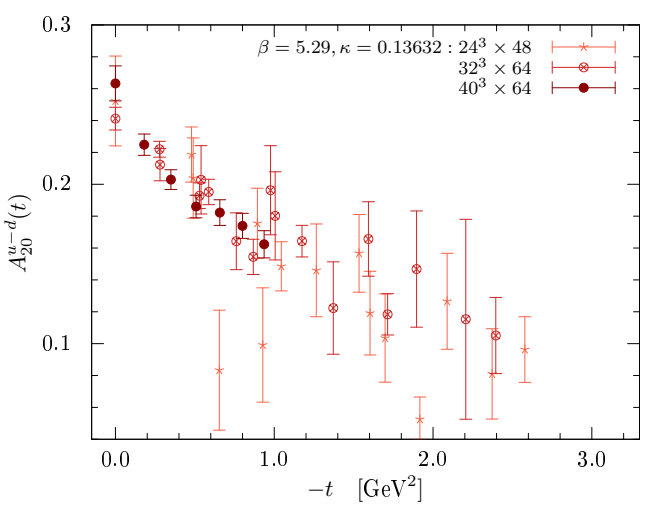

Figure 1: $A_{20}(t)$ for the isovector case for $\beta=$ 5.29 and $\kappa=0.13632$ and for different volumes. urations. The lattice sizes are $24^{3} \times 48,32^{3} \times 64,40^{3} \times 64$ and $48^{3} \times 64$. In particular the latter 

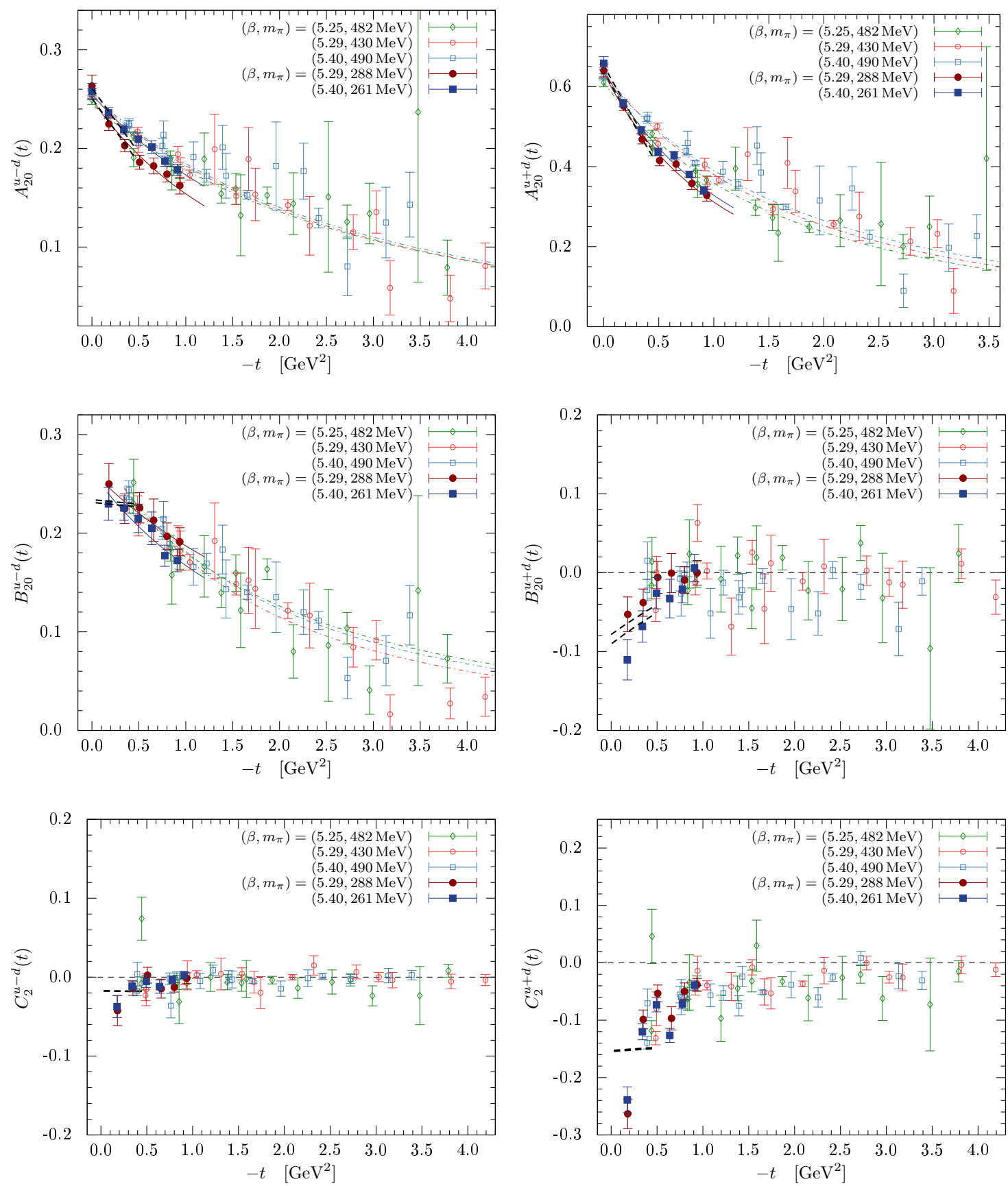

Figure 2: The generalized form factors $A_{20}, B_{20}$ and $C_{2}$ (from top to bottom) vs. momentum transfer $-t$; left for the isovector channel, right for the isosinglet channel. The data is for three lattice spacings and two groups of approximately equal pion masses. If applicable, solid (dashed-dotted) lines represent dipole fits to the data. Dashed lines at low $t$ result from a simultaneous fit of the low-pion-mass data (full symbols) to covariant chiral perturbation theory (see text and also Fig. 3 for more details). 
two provide us with a good signal-to-noise ratio. See, for example, Fig. 1, where data for $A_{20}(t)$ in the isovector channel is shown for the lattice sizes $24^{3} \times 48,32^{3} \times 64$ and $40^{3} \times 64$ at $\beta=5.29$ and $\kappa=0.13632\left(m_{\pi}=287 \mathrm{MeV}\right)$. The number of measurements is 2755,3013 and 1478 , respectively. The advantage of volume averaging is clearly evident as with about half the statistics, the data for the $40^{3} \times 64$ lattice comes with much less statistical noise than that for the $24^{3} \times 48$ lattice. Moreover, Fig. 1 indicates that finite size effects are small, at least at our level of precision.

A selection of all of our GFF data is shown as a function of the momentum transfer $-t$ in Fig. 2. There, the panels from top to bottom display the respective data for $A_{20}(t), B_{20}(t)$ and $C_{2}(t)$. Left panels are for the isovector case, right panels for the isoscalar (without disconnected contributions). For simplicity, only data for five ensembles is shown, which fall into two groups of approximately equal pion mass. For the larger pion mass (430-490 MeV), we have results for three lattice spacings ( $a=0.06,0.07$ and $0.08 \mathrm{fm})$, for the smaller one (260-287 MeV) we can show data for two sets $(a=0.06,0.07 \mathrm{fm})$. For this (admittedly small) range of lattice spacings we observe, however, no systematic dependence on a. Apparently, there is a slight vertical shift in the data for $A_{20}(t<0)$ [and in the opposite direction for $\left.B_{20}(t<0)\right]$ for the lighter sets, but we do not see these shifts for the heavier sets (including

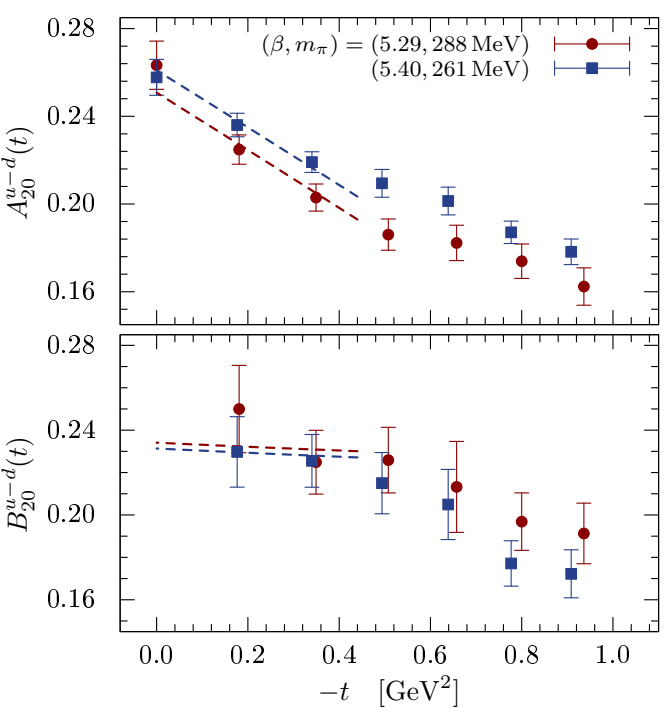

Figure 3: $A_{20}^{u-d}$ (top) and $B_{20}^{u-d}$ (bottom) vs. $-t$ at the pion masses 261 and $288 \mathrm{MeV}$. Dashed lines result from a simultaneous fit of the data (including that for $C_{2}^{u-d}$ ) to expectations from BChPT [14]. those not shown), at least with the available precision. It will be interesting to see how well our forthcoming results at $\beta=5.25, \kappa=0.13620$ (i.e., $a=0.084 \mathrm{fm}, 260 \mathrm{MeV}$ pion mass) fit to these findings.

Similarly, we observe a trend for $A_{20}(t)$ if the pion mass is changed: The low- $t$ dependence of $A_{20}(t)$ gains slope if $m_{\pi}$ is reduced from $430-490 \mathrm{MeV}$ to $260-287 \mathrm{MeV}$. This effect, however, is small and we see no such effect in the data from $500 \mathrm{MeV}$ to $1 \mathrm{GeV}$ pion mass. It thus remains to be seen if this effect at lower $m_{\pi}$ stays or disappears with higher statistics. As above, a further check should become possible as soon as our results at $\beta=5.25$ and $\kappa=0.13620$ are available.

We can confirm though the (notorious) weak $m_{\pi}$ dependence of $A_{20}^{u-d}$ at $t=0$, i.e., of $\langle x\rangle^{u-d}$ (see upper left panel in Fig. 2). From phenomenology one expects $\langle x\rangle^{u-d} \approx 0.16$ at the physical point. So far, however, all available (world) lattice data for $\langle x\rangle^{u-d}$ for pion masses above $200 \mathrm{MeV}$ gives values for $\langle x\rangle^{u-d}$ well above 0.16 , and moreover, almost no signal for a downwards trend towards the physical point is seen (see, e.g., [4] and references therein). From baryon chiral perturbation theory (BChPT), for example, such a trend is expected, but it has not yet been demonstrated (convincingly) on the lattice.

It is however interesting that our data for $|t|<0.4 \mathrm{GeV}^{2}$ indicates an almost linear $t$-dependence for $A_{20}^{u-d}(t)$ and a flattening of the slope for $B_{20}^{u-d}(t)$. This would be consistent with expectations from covariant BChPT at leading-one-loop order [14]. 
In Fig. 2, and in particular in Fig. 3, we show a first attempt of fitting our data to the BChPT expressions for $A_{20}, B_{20}$ and $C_{2}$ as worked out in [14] (see the dashed lines at lower $t$ ). Note that such a fit has to be a combined fit to the data for all three GFFs simultaneously, because the parameter $a_{20}$ enters all of them. The dashed lines in Figs. 2 and 3 refer to such a fit which incorporates only the lighter data sets (full symbols) and points for $|t|<0.44 \mathrm{GeV}^{2}$. Five parameters $\left(a_{20}, b_{20} c_{20}, c_{8}^{r}\right.$ and $\left.c_{12}\right)$ were left free, while the phenomenological value $\langle\Delta x)_{u-d}^{\text {phen }}=0.21$ was used to constrain the coupling $\Delta a_{20}^{v}$. The $m_{\pi}$-dependence of the nucleon mass (entering the BChPT expression for $B_{20}$ and $C_{2}$ ) and the parameter $M_{0}$ were taken from our nucleon mass fits [13].

It turns out that for the isovector case the fit quality is quite good: A reduced $\chi^{2}$-value of about one is reached and the low- $t$ dependence of $A_{20}$ and $B_{20}$ is roughly reproduced; actually, also for $C_{2}$, as only the data point at the smallest $|t|$ falls somewhat below the fitting curve. For the isoscalar case, the same fit works less satisfactorily. The reason might be that BChPT does not work at the pion masses under consideration, or that disconnected contributions are not negligible. The latter are certainly worth to be calculated.

Even though disconnected contributions are still missing, it is interesting to look at the total quark angular momentum

$$
J^{u, d}=\frac{1}{2}\left[A_{20}^{u, d}(0)+B_{20}^{u, d}(0)\right],
$$

to check if it is in the ballpark of expected values. In Fig. 4 we show this data for our lighter sets, that is, for pion masses 261 and $287 \mathrm{MeV}$. Note that for $A_{20}^{u \pm d}$ we have data directly at $t=0$, but not for $B_{20}^{u \pm d}(0)$. However, looking at Fig. 2 one easily sees that the main contribution to $J^{u+d}$ comes from $A_{20}^{u+d}(0)$ and the $t$-dependence of $B_{20}^{u \pm d}$ is compa-

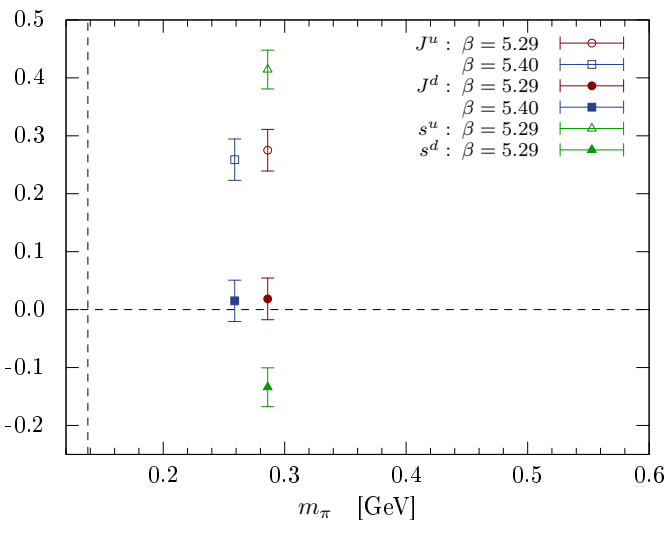

Figure 4: The total quark angular momentum $J$ and spin $s^{q}$ vs. pion mass. rably weak. We therefore approximate $B_{20}^{u \pm d}(0)$ by our data for the smallest $|t|$. This should be perfectly fine for our purposes, given all the other uncertainties and the lack of disconnected contributions. Note that in Fig. 4 we have also included data for the quark spin

$$
s^{q}=\frac{1}{2} \int_{-1}^{1} d x \tilde{H}(x, \xi, 0)=\frac{1}{2} \tilde{A}_{10}^{q}(t=0),
$$

which we obtain from data ${ }^{2}$ for the axial nucleon GFFs $\tilde{A}_{10}^{u-d}$ and $\tilde{A}_{10}^{u+d}$.

If we compare our data in Fig. 4 with that of the LHPC collaboration [15], we find good agreement (albeit their data is for $N_{f}=2+1$ ). We also see the same ordering for the total and orbital $\left(L^{q}=J^{q}-s^{q}\right)$ angular momentum and the quark spin:

$$
\left|J^{d}\right| \ll\left|J^{u}\right|, \quad\left|J^{d}\right| \ll\left|s^{d}\right|, \quad\left|L^{u+d}\right| \ll\left|L^{u}\right|,\left|L^{d}\right| .
$$

It will be interesting to see how this figure changes when data at smaller pion masses becomes available and/or disconnected contributions are included.

\footnotetext{
${ }^{2}$ Unfortunately, there is no data for $\tilde{A}_{10}^{u \pm d}$ at $\beta=5.4$ for these small pion masses, but it will become available soon.
} 


\section{Conclusions}

We have presented an update on our efforts to calculate the generalized form factors for the nucleon. We have restricted ourselves here to the case of $N_{f}=2$ and reported only on results for the GFFs of the energy-momentum tensor $(n=2)$. Due to recent progress on the numerical side we are able to provide data for these GFFs for pion masses down to $260 \mathrm{MeV}$. In particular our lighter sets provide an improvement of the available data for these form factors: Large lattice volumes have allowed us to obtain a very good signal-to-noise ratio, and at low $|t|$ our data starts to fulfill expectations from one-loop BChPT. When comparing our data to that of the LHPC collaboration presented at this conference [15] we see a small vertical offset for the GFF data, but overall agreement for angular momentum and spin. It remains to be seen if this offset is due to the different renormalization procedures of the lattice operators or due to the different $N_{f}$.

The numerical calculations have been performed on the APEmille, apeNEXT systems and PAX cluster at NIC / DESY (Zeuthen, Germany), the IBM BlueGene/L at EPCC (Edinburgh, UK), the BlueGene/P (JuGene) and the Nehalem Cluster (JuRoPa) at NIC (Jülich, Germany), and the SGI Altix and ICE 8200 systems at LRZ (Munich, Germany) and HLRN (Berlin-Hannover, Germany). We have made use of the Chroma software suite [16]. The BlueGene codes were optimised with Bagel [17]. This work has been supported in part by the DFG (SFB/TR 55, Hadron Physics from Lattice QCD) and the EU under grants 238353 (ITN STRONGnet) and 227431 (HadronPhysics2). A.St acknowledges support by the European Reintegration Grant (FP7-PEOPLE-2009-RG, No.256594). JZ is supported by the University of Adelaide and the Australian Research Council through a Future Fellowship (FT100100005).

\section{References}

[1] A. Belitsky and A. Radyushkin Phys.Rept. 418 (2005) 1-387, [hep-ph/ 0504030 ].

[2] M. Göckeler et al. Phys.Rev. D82 (2010) 114511, [1003. 5756].

[3] X.-D. Ji J.Phys. G24 (1998) 1181-1205, [hep-ph/ 9807358$].$

[4] P. Hägler Phys.Rept. 490 (2010) 49-175, [0912.5483].

[5] QCDSF Collaboration, M. Göckeler et al. Phys.Rev.Lett. 92 (2004) 042002, [hep-ph/ 0304249 ].

[6] LHPC + SESAM Collaboration, P. Hägler et al. Phys.Rev. D68 (2003) 034505, [hep-lat/0304018].

[7] LHPC + SESAM Collaboration, P. Hägler et al. Phys.Rev.Lett. 93 (2004) 112001, [hep-lat/0312014].

[8] LHPC Collaboration, P. Hägler et al. Phys.Rev. D77 (2008) 094502, [0 705 . 4295].

[9] QCDSF/UKQCD Collaboration, D. Brömmel et al. Phys.Rev.Lett. 101 (2008) 122001, [0708.2249].

[10] LHPC Collaboration, J. Bratt et al. Phys.Rev. D82 (2010) 094502, [1001 . 3620 ].

[11] C. Alexandrou et al. Phys.Rev. D83 (2011) 114513, [1104.1600].

[12] S. Collins et al. Phys.Rev. D84 (2011) 074507, [1106.3580].

[13] QCDSF Collaboration, G. Bali et al. in preparation.

[14] M. Dorati, T. A. Gail, and T. R. Hemmert Nucl.Phys. A798 (2008) 96-131, [nucl-th/ 0703073 ].

[15] S. Syritsyn et al. PoS(Lattice 2011)178, [1111.0718].

[16] SciDAC + LHPC + UKQCD Collaboration, R. G. Edwards and B. Joo Nucl.Phys.Proc.Suppl. 140 (2005) 832, [hep-lat/0409003].

[17] P.A. Boyle, http://www.ph.ed.ac.uk/ paboyle/bagel/Bagel.html (2005). 